\title{
Dynamic behavior of the vortex ring formed on a butterfly wing
}

\author{
Masaki Fuchiwaki • Taichi Kuroki • \\ Kazuhiro Tanaka $\cdot$ Takahide Tababa
}

Received: 12 April 2011 /Revised: 17 December 2012/ Accepted: 18 December 2012/Published online: 4 January 2013

(C) The Author(s) 2013. This article is published with open access at Springerlink.com

\begin{abstract}
Micro-air-vehicles (MAVs) and micro-flight robots that mimic the flight mechanisms of insects have attracted significant attention in recent years. A number of MAVs and micro-flight robots that use various devices have been reported. However, these robots were not practical. One of the reasons for this is that the flying mechanism of insects has not yet been clarified sufficiently. In particular, the dynamic behavior of the vortex formed on the insect wing and its growth process have not been clarified. The purpose of the present study is to clarify the dynamic behavior and the detailed structure of the vortices of the flapping butterfly wing. The authors conducted a particle image velocimetry measurement around the flapping butterfly wing of Cynthia cardui and Idea leuconoe and investigated the vortex structure of the wing and its dynamic behavior. A vortex ring is formed over the butterfly wings when the wings flap downward to the bottom dead position. The vortex ring then passes over the butterfly completely and grows until reaching the wake at the bottom dead position. The vortex ring is formed over the
\end{abstract}

\footnotetext{
M. Fuchiwaki $(\bowtie) \cdot$ T. Kuroki · K. Tanaka

Department of Mechanical Information Science and Technology,

Kyushu Institute of Technology, 680-4 Kawazu, Iizuka-shi,

Fukuoka 820-8502, Japan

e-mail: futiwaki@mse.kyutech.ac.jp

T. Kuroki

e-mail: kuroki@vortex.mse.kyutech.ac.jp

K. Tanaka

e-mail: kazuhiro@mse.kyutech.ac.jp

T. Tababa

Department of Mechanical Engineering, Kagoshima National

College of Technology, 1460-1 Shinkou, Kirishima-shi,

Kagoshima 899-5193, Japan

e-mail: tabata@kagoshima-ct.ac.jp
}

wings regardless of the type of butterfly, although the scale of the vortex ring varies with the butterfly type.

\section{Introduction}

Butterflies fly by combining wing flapping and gliding efficiently and have beautiful flight patterns. Moreover, the butterfly excels in rapid acceleration and turning. As a result of recent developments in micro-electro-mechanical systems (MEMS), micro-air-vehicles (MAVs) and microflight robots that mimic the flight mechanisms of insects have been attracting significant attention. These technologies are developed for use in search and rescue operations in areas having a high risk of secondary disasters, maintenance work in construction interplanetary information collection, and the monitoring of security risks. A number of MAVs and micro-flight robots that use various devices have been reported. Tanaka et al. (2005) developed an externally powered butterfly-type ornithopter (BTO) to investigate butterfly flight. Their BTO has a mass of $0.4 \mathrm{~g}$, a wingspan of $140 \mathrm{~mm}$, and flapping frequency of $10 \mathrm{~Hz}$. They visualized the flow field around the BTO and revealed that free-body motion caused a stable attachment of leading edge vortices. Moreover, they considered the deformation of the flapping wing and compared two types of venation in order to clarify the function of the inner veins. They clarified the effect of the inner veins on the flight performance (Tanaka and Shimoyama 2010). Zaeem et al. (2006) investigated designs of flapping mechanisms that allow high-amplitude, high-frequency flapping. They reported that these mechanism use springs and passive flapping, a concept inspired by the study of the wing motion of insects and hummingbirds, and these mechanisms simulate the transverse bending effect of insect 
wings to achieve high flapping amplitudes. Singh and Chopra (2008) addressed the aerodynamics of insect-based, biomimetic, flapping wings in hover. They measured the thrust generated by a number of wings mounted on a flapping-pitching mechanism for a number of wing and stroke parameters. However, these robots were not practical. One of the reasons for this is that the flying mechanism of insects has not yet been clarified sufficiently.

A number of studies on the mechanism of butterfly flight have been carried out in recent years. Sunada et al. (1993) observed a butterfly during takeoff by a flying observation experiment involving Pieris melete and reported the characteristics of behaviors of the wings and the forces that a butterfly generates. Dickinson et al. (1999) reported three important aerodynamic mechanisms, namely, delayed stall, rotational circulation, and wake capture. Senda et al. (2004) analyzed motions and measured the forces of Parantica sita through a flying observation experiment. In addition, they simulated the motions of its wings by numerical calculation. Lee et al. (2008) conducted a detailed numerical simulation to investigate the aerodynamic characteristics of unsteady force generation by a two-dimensional insect flapping motion under a forward flight condition. Moreover, a number of recent studies have examined the flow field around an insect wing. Dickinson and Gotz (1996) used flow visualization and instantaneous force measurements of tethered fruit flies, Drosophila melanogaster, to study the dynamics of force generation during flight and reported an animated reconstruction of the vortex loop formed during the downstroke and ventral flap. Bomphrey et al. (2005) carried out the first digital particle image velocimetry (DPIV) around the wings of an insect, Manduca sexta, and reported the relationship between a leading edge vortex (LEV) structure and force production. Subsequently, Bomphrey et al. (2006) reported what broadly resembled an elliptical vortex loop around a tethered Tobacco Hawkmoth, Manduca sexta. Yuan and Gong (2008) clarified the detailed three-dimensional flow structures and evolution of the LEV on a flapping wing in the hovering condition. Bomphrey et al. (2009) applied classical smoke line visualization techniques to analyze the aerodynamic mechanisms of free-flying bumblebees and reported the independent LEV on each wing. However, the dynamic behavior of the vortex formed on the insect wing and its growth process have not yet been clarified.

The present authors conducted a flight observation experiment involving Cynthia cardui and clarified the behaviors of its wings in flight. By spatial evaluation of the wing in flapping flight, the authors have clarified that the flapping angle, lead-lag angle, and feathering angle of the butterfly have periodicity. Moreover, we have clarified that the wing deforms elastically not only in the wing chordwise direction but also in the wing spanwise direction
(Fuchiwaki and Tanaka 2006). Based on these results, we developed a flapping-wing robot without tail wings, which is similar to a real butterfly (Fuchiwaki et al. 2009). The mass of the robot, including the lithium-ion battery and the motor, is $1.9 \mathrm{~g}$, and the wing span is $240 \mathrm{~mm}$. The wing chord is $80 \mathrm{~mm}$. The robot can fly stably for approximately $20 \mathrm{~min}$. The elastic deformation of the wings was found to be an important parameter for stable flight, and we focused on the flow field around the wings created by the flapping motion and its elastic deformation. Furthermore, through a visualization experiment using particles, we qualitatively evaluated the two-dimensional vortical structures that formed around a butterfly wing and clarified that a pair of large-scale vortices is formed on the top face of the wing in the span direction (Fuchiwaki and Tanaka 2008). However, the dynamic behavior and the detailed structure of the vortices of the butterfly wing have not yet been sufficiently clarified.

The purpose of the present study is to clarify the dynamic behavior and the detailed structure of the vortices of the flapping butterfly wing under the fixed condition. The authors conducted a PIV measurement around the wing of Cynthia cardui and Idea leuconoe and investigated the vortex structure of the wing and its dynamic behavior. The dynamic behavior of the butterfly wings under the fixed condition is similar to those in take-off flight. In the future, the results of the present study will be helpful for developing the take-off mechanism of the flapping-wing robot, such as the flapping motions and materials of the wings. Moreover, the results of the present paper will provide a fundamental understanding of the vortex structure around the butterfly wing and will help to clarify the flow field around butterfly wings in free flight.

\section{Experiments}

\subsection{Butterflies considered in the present experiments}

The butterflies considered in the present study are Cynthia cardui and Idea leuconoe, as shown in Fig. 1. The wing span length $l$, wing chord length $c$, aspect ratio of the wing $A R$, wing area $A$, mass $m$, wing load $m g / A$, flapping frequency of the wing $f$, and average wing tip velocity $V$ of two butterflies in our experiments and our flapping-wing robot are listed in Table 1. The wing span is defined as the maximum wing length, as shown in Fig. 1, and the wing chord is calculated as the wing area divided by the wing span (Marden 1987). The flapping frequencies of the wings in both butterflies are low compared to those of the hawkmoth, $26.1 \mathrm{~Hz}$ (Van Den Berg and Ellington 1997) and bumblebee, $180 \mathrm{~Hz}$ (Bomphrey et al. 2009). In particular, the flapping frequency of Idea leuconoe is very 


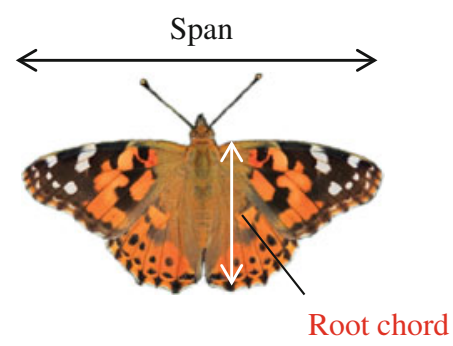

(a) Cynthia cardui

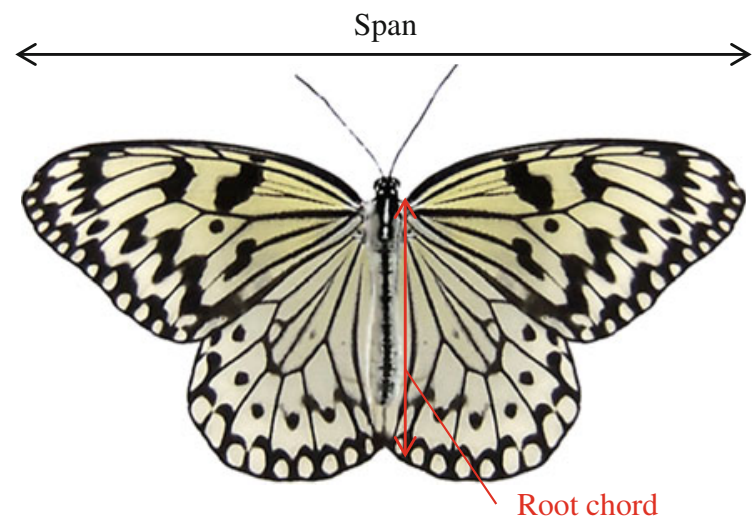

(b) Idea leuconoe

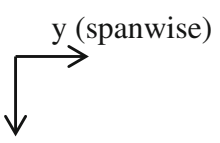

$\mathrm{x}$ (chordwise)

Fig. 1 Butterflies considered in the present study

low. Therefore, the vortex flow and its dynamic behavior generated by the flapping wings of the butterfly are expected to be important for generating the aerodynamic forces required for flight.

\subsection{PIV measurement system}

The PIV measurement equipment consists of an acrylic container, a high-speed camera, a 5-W Nd:YAG continuous-wave laser [wavelength of $532(\mathrm{~nm})$ ], and a butterfly, as shown in Fig. 2. The test section in the acrylic container is $900(\mathrm{~mm})$ in length, $900(\mathrm{~mm})$ in width, and $900(\mathrm{~mm})$ in depth. Expancel (Kanomax), which has a diameter of approximately $10 \mu \mathrm{m}$ and a specific gravity of 0.7 , was used as a tracer particle. The acrylic container is filled with tracer particles in order to keep an average of approximately eight particle images per region (Adrian 1991). The tracer particles are floated by an air duster in the test section, and the PIV measurement is started by dropping the tracer particle very slowly. In the experiments, the legs of the butterfly were fixed to a shaft in the test section without interfering with its wing motion. Two-dimensional images

Table 1 Dimensions of the butterflies considered in the present study

\begin{tabular}{lccc}
\hline & $\begin{array}{l}\text { Cynthia } \\
\text { cardui }\end{array}$ & $\begin{array}{l}\text { Idea } \\
\text { leuconoe }\end{array}$ & $\begin{array}{l}\text { Flapping-wing robot } \\
\text { (Fuchiwaki et al. 2009) }\end{array}$ \\
\hline$l(\mathrm{~mm})$ & 65 & 140 & 240 \\
$c(\mathrm{~mm})$ & 11.3 & 23.8 & 52.8 \\
$A R$ & 5.8 & 5.9 & 4.5 \\
$A\left(\mathrm{~mm}^{2}\right)$ & 737 & 3,333 & 12,667 \\
$m(\mathrm{~g})$ & 0.16 & 0.40 & 1.90 \\
$m g / A\left(\mathrm{~N} / \mathrm{m}^{2}\right)$ & 2.17 & 1.20 & 1.50 \\
$f(\mathrm{~Hz})$ & 13.0 & 6.0 & 10.0 \\
$V(\mathrm{~m} / \mathrm{s})$ & 0.42 & 0.42 & 1.20 \\
\hline
\end{tabular}

around the flow fields of the butterfly wings were captured by the high-speed camera (2,000 fps).

The PIV images were processed using Davis 8.0 software from LaVision $\mathrm{GmbH}$, which incorporates three-pass cross-correlation to realize high accuracy and high resolution of the derived velocity and vorticity fields (Hart 2000; Bomphrey et al. 2005, 2006). A primary correlation window of $64 \times 64$ pixels was selected with a sub-correlation window of $32 \times 32$ pixels and a search radius of $9 \times 9$ pixels with a $50 \%$ overlap. This typically yielded $64 \times 64$ velocity vectors per PIV image and corresponded to a spatial resolution of $0.07 \mathrm{~mm} \times 0.07 \mathrm{~mm}$ over an imaging area of $72 \mathrm{~mm} \times 72 \mathrm{~mm}$ (Cynthia cardui) and to a spatial resolution of $0.23 \mathrm{~mm} \times 0.23 \mathrm{~mm}$ over an imaging area of $236 \mathrm{~mm} \times 236 \mathrm{~mm}$ (Idea leuconoe). The velocity vector and vorticity fields were calculated using this software. Based on error analysis (Hart 2000; Lawson and Davidson 2001; Ansari et al. 2009), the error in the PIV data is estimated to be in the range of $\pm 0.0468(\mathrm{~m} / \mathrm{s})$.

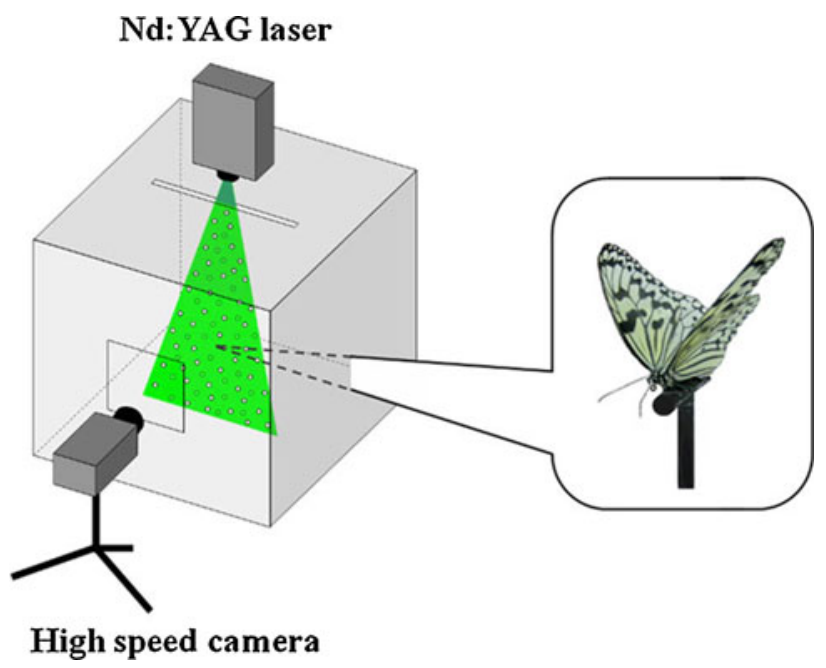

Fig. 2 PIV measurement equipment 
An Nd:YAG laser irradiated the wing in the spanwise planes and the chordwise planes, as shown in Fig. 3a, b, respectively. The irradiation positions were 12 spanwise planes, including the leading edge, the center of the wing chord, the trailing edge, and the wake, and four chordwise planes, including the wing tip, the center of the semi-span, the basic point of the wing, and the body. Moreover, in order to clarify the three-dimensional vortex structure over the wing, the Nd:YAG laser irradiated the wing in the oblique plane with respect to the wing spanwise plane and at the bottom of the butterfly, as shown in Fig. 4a, b, respectively.

Figure 5a, b show the traces of the flapping wing tips of Cynthia cardui and Idea leuconoe, respectively, the legs of which were fixed to a shaft. Moreover, Fig. 6a, b show the traces of the flapping wing tip of Cynthia cardui and Idea leuconoe during take-off flight, respectively. The horizontal and vertical axes indicate the lead-lag angle [rad.] and the flapping angle [rad.], respectively. The red and blue lines show the traces of the flapping wing tips in the flap-up and flap-down motions, respectively. The dynamic behaviors of the flapping wings of the butterflies under the fixed condition are similar to those in take-off flights, and the motions of the flapping wings are strongly periodic in both cases. Therefore, the flow field around the butterfly is also expected to change periodically. In the present study, we obtain a two-dimensional flow field at each point, as shown in Fig. 3a, b. In addition, we compare the results for the flapping angle and clarify three-dimensional vortex structure over the butterfly wings.

\section{Results and discussions}

\subsection{A pair of vortices formed on the wing}

Figure 7 shows the PIV measurement results around the butterfly wing of Idea leuconoe, with the wings at the near bottom dead position. A pair of vortices is clearly observed over the wings. This pair of vortices is formed between the wings in the downward flapping motion and develops over the wing surface.

In the present study, a proper vortex identification criterion is critical. Bomphrey et al. (2005) defined the vortex region as a region of high vorticity with a centered streamline. Yuan and Gong (2008) defined a vortex using the second invariant of the velocity gradient tensor. In the present study, the vorticity, $\omega=0.05(1 / \mathrm{s})$, was defined as

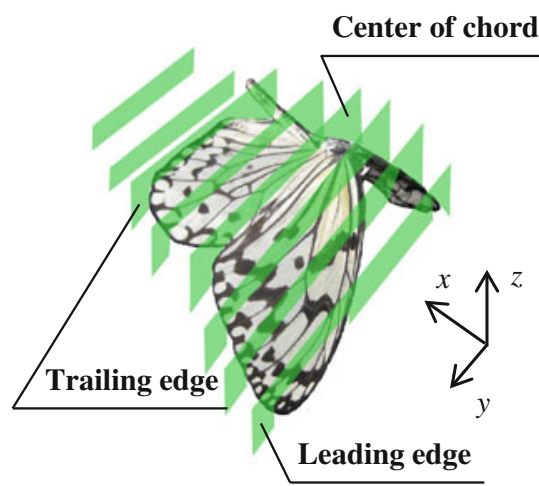

(a) Spanwise planes

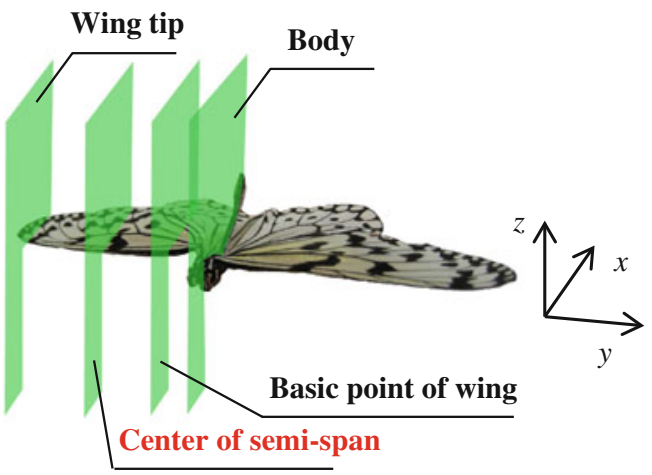

(b) Chordwise planes

Fig. 3 Irradiation positions of the Nd:YAG laser in the wing spanwise and chordwise planes

Fig. 4 Irradiation positions of the Nd:YAG laser in the wing oblique plane with respect to the wing spanwise plane and at the bottom of the butterfly

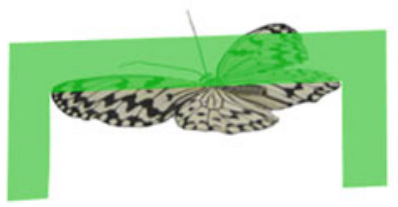

(a) Oblique plane

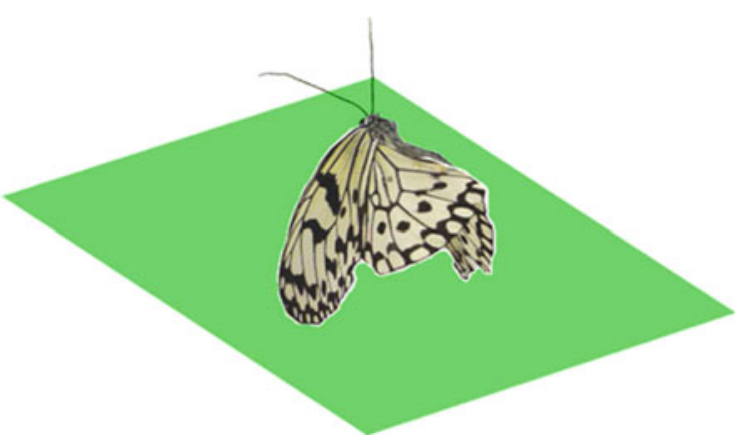

(b) Bottom of butterfly 


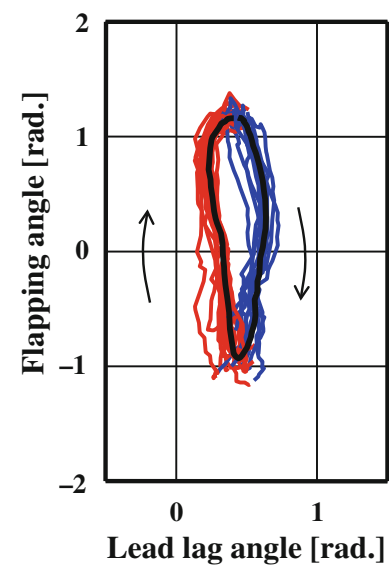

(a) Cynthia cardui

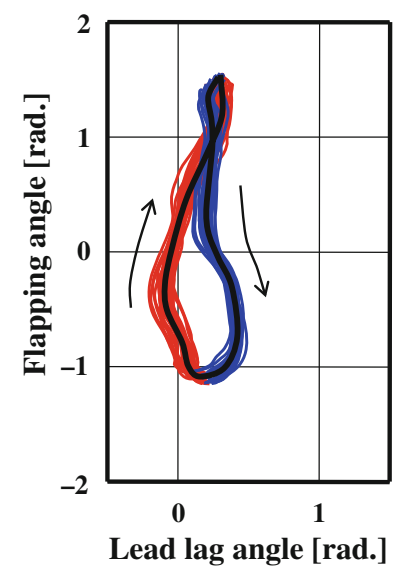

(b) Idea leuconoe
Fig. 5 Traces of the flapping wing tips of Cynthia cardui and Idea leuconoe

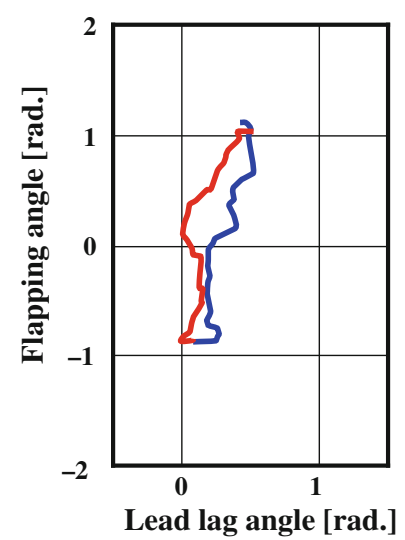

(a) Cynthia cardui

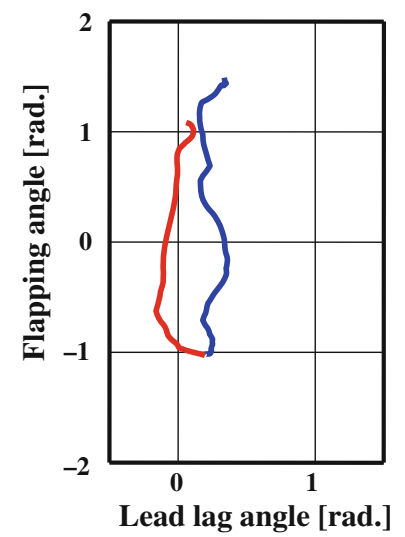

(b) Idea leuconoe
Fig. 6 Traces of the flapping wing tip of Idea leuconoe performing take-off flight

the threshold, and the area having a vorticity of over 0.05 $(1 / s)$ was defined as a vortex in the PIV measurement results. Figure $8 \mathrm{a}, \mathrm{b}$ show the vorticity contours over 0.05 $(1 / \mathrm{s})$ and the velocity vectors in relation to the vorticity over $0.05(1 / \mathrm{s})$, respectively, in the flow field over the butterfly wings in downward flapping. We evaluated the pair of vortices in each section in the spanwise planes and investigated the detailed vortex structure on the total area of the wings.

Figure 9 shows the velocity vectors in relation to the vorticity over $0.05(1 / \mathrm{s})$ around the downward flapping wings of Idea leuconoe. Figure 9a through d show the results for the leading edge, the center of the chord, the trailing edge, and the wake, respectively.

A pair of vortices was confirmed over the wings at all points. This result indicates that tube-type vortices formed over each wing. Moreover, the velocity vectors at the

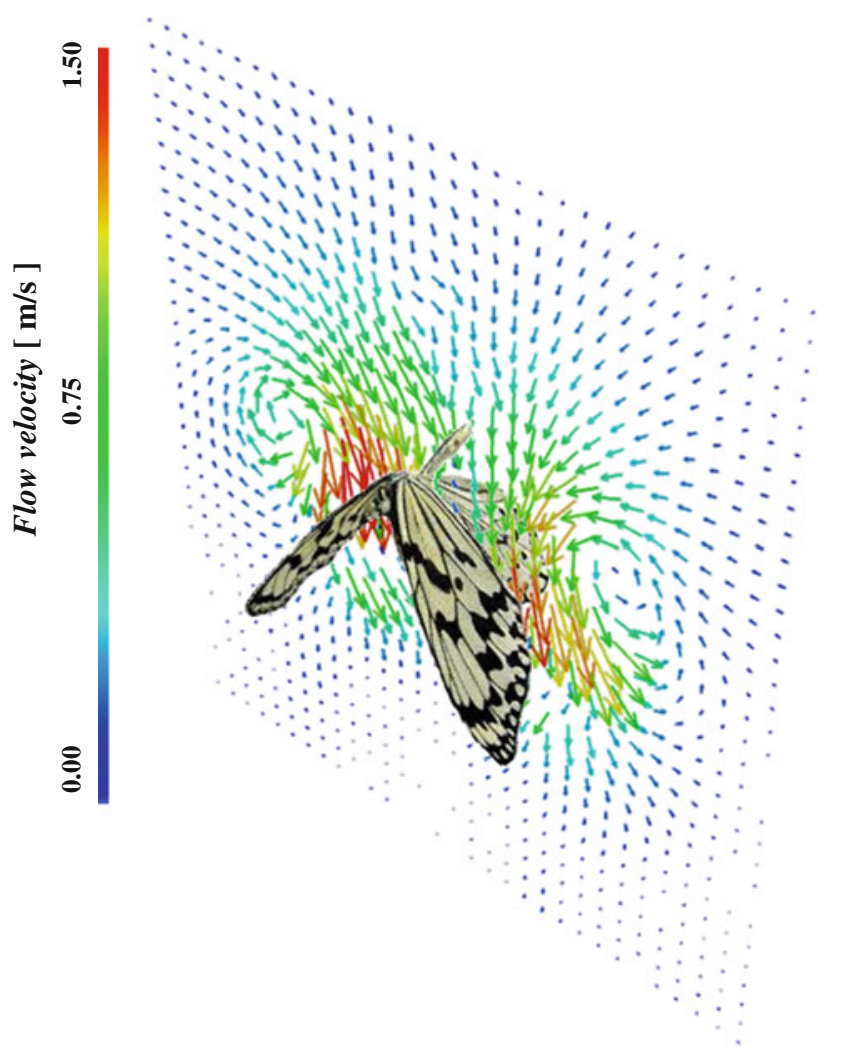

Fig. 7 Velocity vectors around the butterfly wing of Idea leuconoe with the wings at the near bottom dead position

leading edge were very fast, as shown in Fig. 9a. However, the velocity vectors from the trailing edge to the wake became slow, as shown in Fig. 9c, d.

Figure 10 shows the velocity vectors in relation to the vorticity over $0.05(1 / \mathrm{s})$, around the wings of Idea leuconoe at the near bottom dead position. Figure 10a-d show the results at the leading edge, the center of the chord, the trailing edge and the wake, respectively. As in Fig. 9, a pair of vortices was formed over the wings even at the near bottom dead position. A tube-type vortex was found to form over the wings at the near bottom dead position, as shown by the pair of vortices that was confirmed at all points. Moreover, as shown in Fig. 10a, not only the scales but also the velocities of the vortices formed at the leading edge were smaller than those that formed at the trailing edge at the near bottom dead position, as shown in Fig. 10c. Based on these results, the vortices formed on the wings were found to grow from the leading edge to the trailing edge in downward flapping motion. Moreover, the scale of the vortices and their vorticity increases toward the bottom dead position of the flapping motion. Therefore, the vortices were expected to develop spirally in the wing chordwise direction. These results were confirmed using Cynthia cardui. 
Fig. 8 Vorticity contours over $0.05(1 / \mathrm{s})$ and velocity vectors in relation to the vorticity over $0.05(1 / \mathrm{s})$ over the butterfly wings in downward flapping

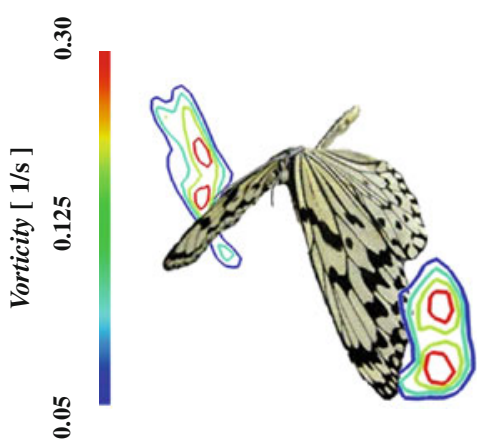

(a) Vorticity contours

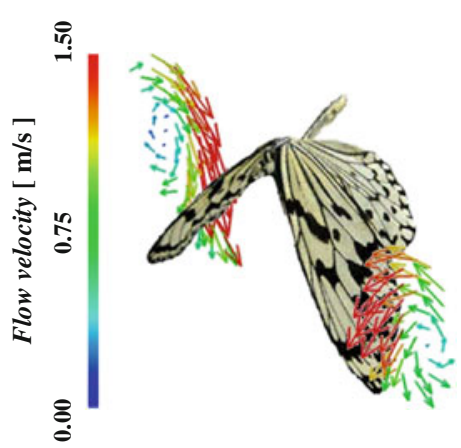

(b) Velocity vectors

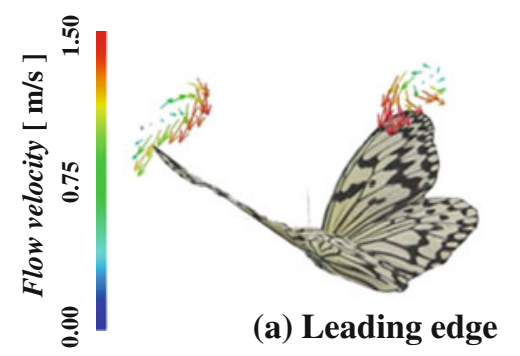

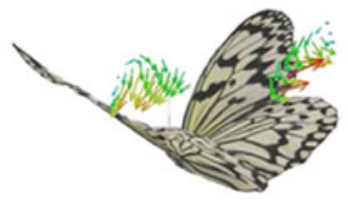

(b) Chord center

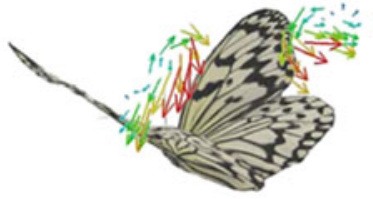

(c) Trailing edge

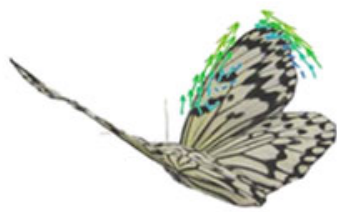

(d) Wake

Fig. 9 Velocity vectors in relation to the vorticity over $0.05(1 / \mathrm{s})$ around the downward flapping wings of Idea leuconoe
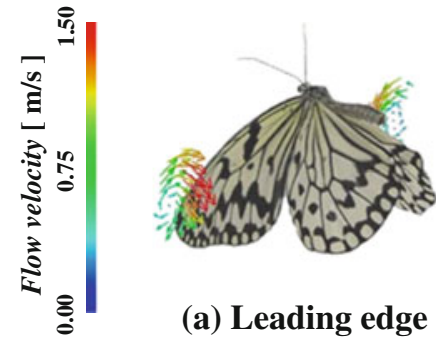

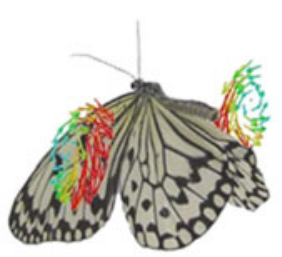

(b) Chord center

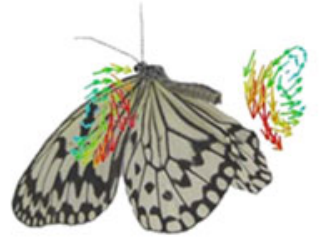

(c) Trailing edge

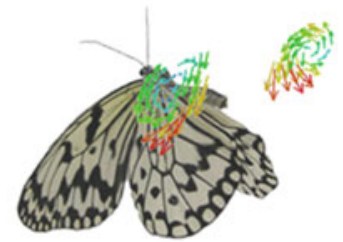

(d) Wake

Fig. 10 Velocity vectors in relation to the vorticity over $0.05(1 / \mathrm{s})$ around the wings of Idea leuconoe at the near bottom dead position

\subsection{Leading edge vortices on the wing}

Figure 11 show the velocity vectors in relation to the vorticity over $0.05(1 / \mathrm{s})$ around the leading edge of the wings of Idea leuconoe in downward movement. Figure 11a-c show the results at the near top dead position, the horizontal position, and the near bottom dead position, respectively.

As shown in Fig. 11a, the leading edge vortex (LEV) is shed when the butterfly flaps its wings downward from the top dead position. The LEV is shed even at the base of the wings and the head of butterfly in the horizontal position. Based on these results, a tube-type vortex was found to form in the spanwise direction, similar to that in the chordwise direction, due to the LEV being shed at all points in the chordwise planes. These results were also confirmed using Cynthia cardui.

\subsection{A vortex ring on the wing}

Figures 12a, b show the iso-surfaces of $\omega^{\prime}=7.69 \times 10^{-3}$ for Cynthia cardui and $8.33 \times 10^{-3}$ for Idea leuconoe, respectively. The non-dimensional vorticity $\omega^{\prime}$ is obtained as follows:

$\omega^{\prime}=\frac{\omega(l / 2)}{v^{\prime}}$,

where $\omega$ is the vorticity obtained by PIV measurement and $v^{\prime}$ is the average wing tip velocity. These results are for the downward flapping motion.

Based on the results for the iso-surface of the nondimensional vorticity, tube-shaped vortices (hereinafter referred to as tube-type vortices) were found to have formed on the wing in the wing chordwise direction in both cases. In Cynthia cardui, as shown in Fig. 12a, tube-type 


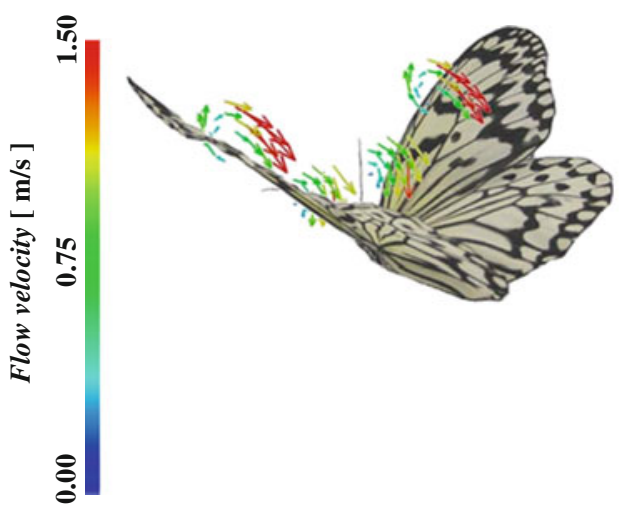

(a) Near top dead position

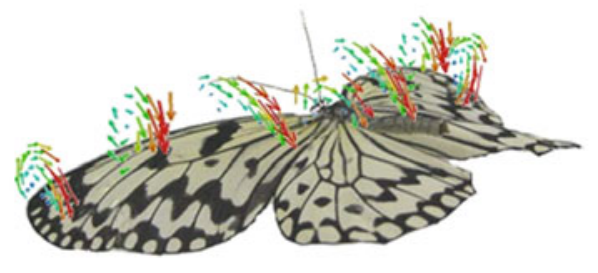

(b) Horizontal position

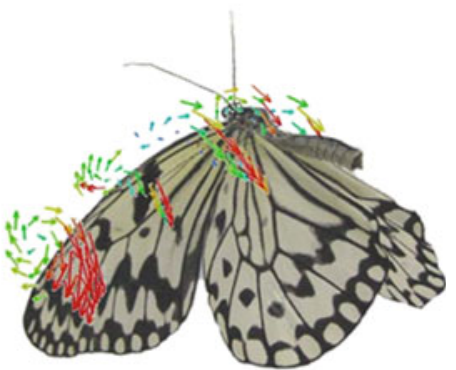

(c) Near bottom dead position

Fig. 11 Velocity vectors in relation to the vorticity over $0.05(1 / \mathrm{s})$ around the leading edge of the wings of Idea leuconoe in downward movement

Fig. 12 Iso-surfaces of $\omega^{\prime}=7.69 \times 10^{-3}$ for Cynthia cardui and $8.33 \times 10^{-3}$ for Idea leuconoe in the wing chordwise direction for downward flapping motion of the wings

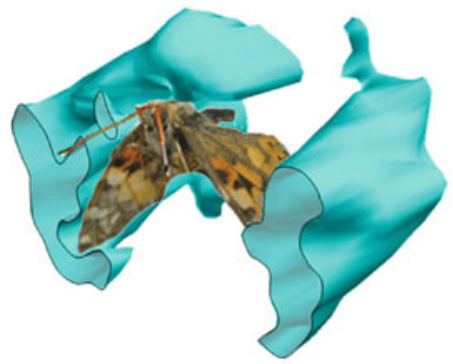

(a) Cynthia cardui

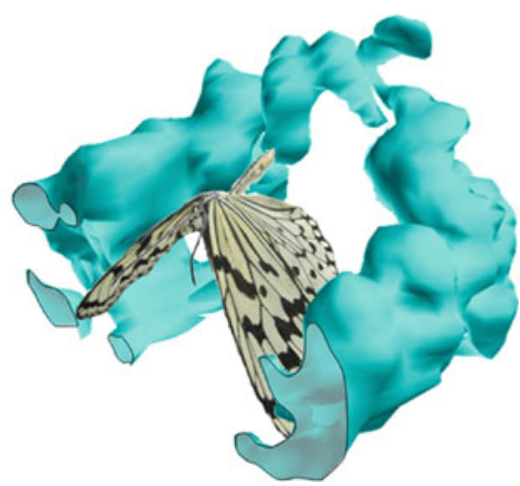

(b) Idea leuconoe vortices clearly formed over the wings. Moreover, these tube-type vortices are large compared with the wings and become linear. Similarly, tube-type vortices formed over the wings of Idea leuconoe, as shown in Fig. 12b. Although the threshold of the iso-surface in Idea leuconoe is high compared with that in Cynthia cardui, the vortices are clear. Moreover, the tube-type vortices formed on each of the wings are connected behind one chord length. In Cynthia cardui, the wing load is approximately two times larger than that of Idea leuconoe, and the flapping frequency of the wings is twice as large as that of Idea leuconoe. Thus, the vorticity of the vortices shed from the wings is high, and tube-type vortices are formed linearly. Therefore, the tube-type vortices that formed over each of the wings did not join behind the wings. However, these vortices are expected to join further downstream.

Figure 13 shows the iso-surfaces in the wing spanwise direction. These are the results for the downward flapping motion. As in Fig. 12, a tube-type vortex formed on the wings, this time in the wing spanwise direction. The tubetype vortices formed near the head is small, but the vortices grow toward the wing tip. These results were also confirmed using Cynthia cardui. These tube-type vortices formed in the wing spanwise direction and are thought to be similar to the Class 2 LEV obtained by Bomphrey et al. (2005).

Figure 14 shows the velocity vectors in relation to the vorticity over $0.05(1 / \mathrm{s})$ around the wing tip, irradiated in Fig. $4 \mathrm{a}$, of Idea leuconoe in downward movement. A vortex is clearly observed over the wing tip. Based on the results shown in Figs. 12, 13, and 14, the tube-type vortices formed in the wing chordwise and spanwise directions were found to be connected. Moreover, Fig. 15 shows the velocity vectors at the bottom of Idea leuconoe in wing downward movement. A ring-shaped flow appears below the butterfly. The connection of the tube-type vortices formed on both wings in the wing chordwise directions behind the butterfly, as discussed in Fig. 12, can be explained by this result.

Figure 16 shows the pattern diagrams of the vortex on the wings of Idea leuconoe obtained from the iso-surfaces of vorticity. Figures 16a through h show the results for the region near the top dead position, moving downward, moving further downward, at the bottom dead position, moving upward, moving further upward, near the top dead position, and at the top dead position, respectively. 


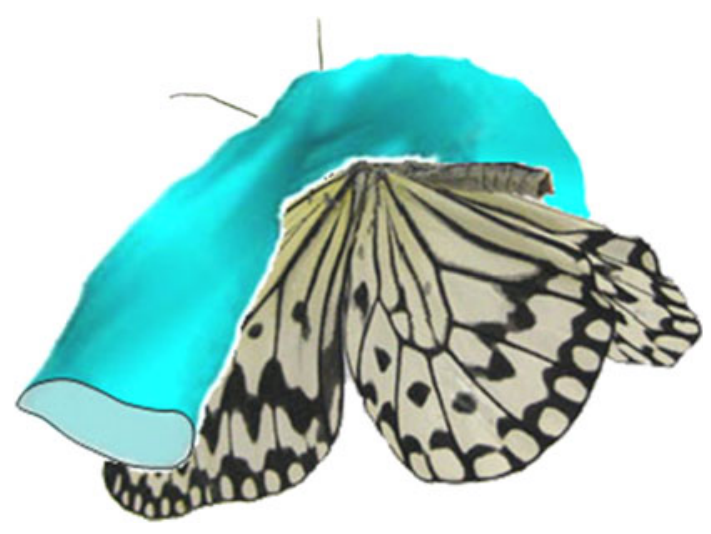

Fig. 13 Iso-surfaces in the wing spanwise direction

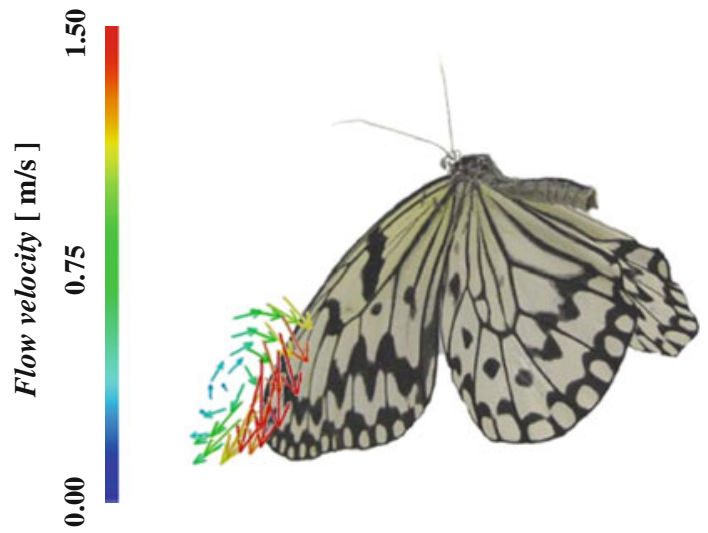

Fig. 14 Velocity vectors in relation to the vorticity over $0.05(1 / \mathrm{s})$ around the wing tip of Idea leuconoe in downward movement

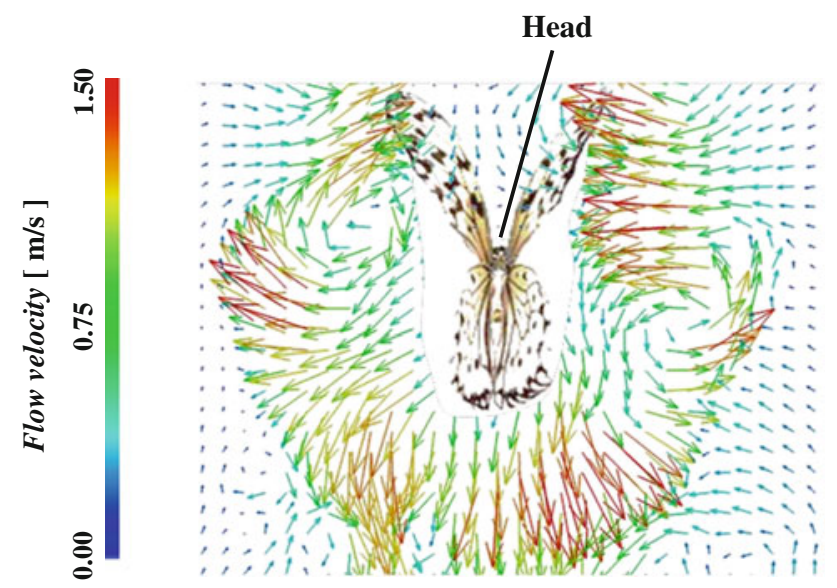

Fig. 15 Velocity vectors at the bottom of the butterfly of Idea leuconoe in wing downward movement

A vortex ring has begun to form when the wings flap downward, as shown in Fig. 16a, and has formed completely over the wings when the wings flap further downward, as shown in Fig. 16b. The vortex ring develops over the wings when the wings flap further downward, as shown in Fig. 16c, and then passes over the butterfly completely and grows until reaching the wake at the bottom dead position, as shown in Fig. 16d. The scale of the front of the vortex ring is large when the wings flap downward, as shown in Fig. 16b, and the scale of the middle of the vortex ring becomes larger with increasing flapping motion, as shown in Fig. 16c. In other words, the vortex ring develops over the wing while growing from the leading edge toward the trailing edge, and the back of the vortex ring rises and elongates because of the growth induced by the vortex ring. Moreover, another vortex ring has begun to form when the wings flap upward, as shown in Fig. 16e. When the wings flap upward to the top dead position, as shown in Fig. 16f, g, the vortex ring has formed completely over the wings. After that, the vortex ring passes over the butterfly completely and grows until reaching the wake, as shown in Fig. 16h. However, the vortex ring formed in flapping upward is smaller than that formed in flapping downward. This is thought to be due to the elastic deformation in the wings. Butterfly wings have numerous veins of various diameters and stiffnesses. Consequently, the elastic deformation in flapping upward is different from that in flapping downward.

Figure 17 shows the pattern diagrams of the vortex on the wings of Cynthia cardui obtained by the iso-surfaces of vorticity. Figure 17a through $\mathrm{h}$ show the results for the region near the top dead position, moving downward, moving further downward, at the bottom dead position, moving upward, moving further upward, near the top dead position, and at the top dead position, respectively.

Similarly, a vortex ring has begun to form when the wings flap downward, as shown in Fig. 17a, and has formed completely over the wings when the wings flap downward to the bottom dead position, as shown in Fig. 17b, c. The vortex ring then passes over the butterfly completely and grows until reaching the wake at the bottom dead position, as shown in Fig. 17d. For upward flapping, another vortex ring has begun to form, as shown in Fig. 17e. When the wings flap upward to the top dead position, as shown in Fig. 17f, g, the vortex ring has formed completely over the wings. After that, the vortex ring passes over the butterfly completely and grows until reaching the wake, as shown in Fig. 17h, as in the case of Idea leuconoe. The vortex ring is formed over the wings regardless of the type of butterfly, although the scale of the vortex ring varies with the butterfly type. In Cynthia cardui, the vortices that developed in the spanwise planes are larger than those that developed in the chordwise planes because the flapping frequency is high and tube-type vortices become linear. Although the difference in the vortex ring scale in flapping upward and downward is not clear, the scale of the vortex ring formed in flapping upward is 
Fig. 16 Pattern diagrams of the vortex on the wings of Idea leuconoe for downward flapping motion of the wings

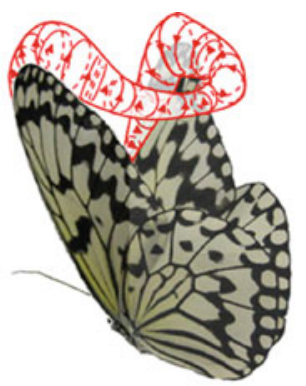

(a) Near top dead position

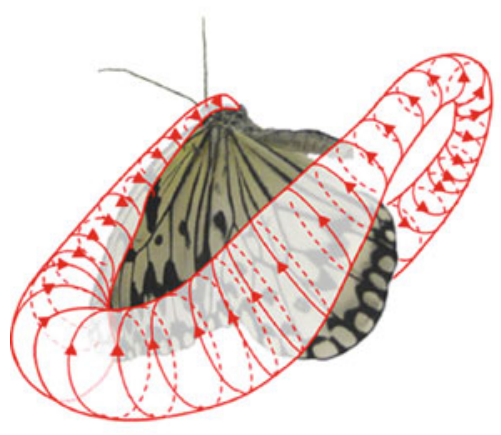

(c) Moving further downward

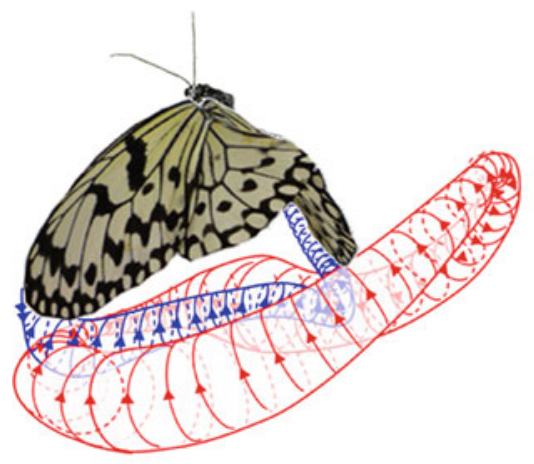

(e) Moving upward

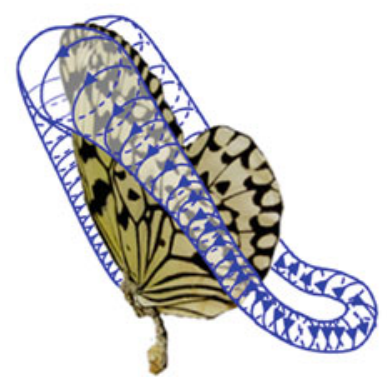

(g) Near top dead position

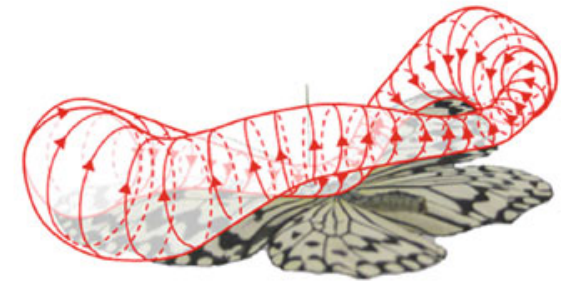

(b) Moving downward

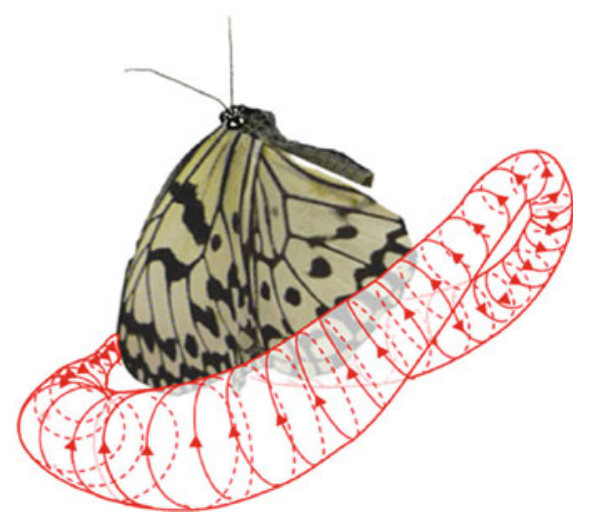

(d) Bottom dead position
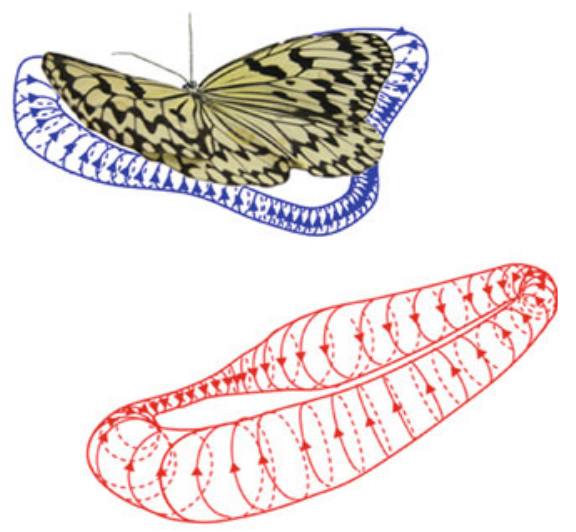

(f) Moving further upward

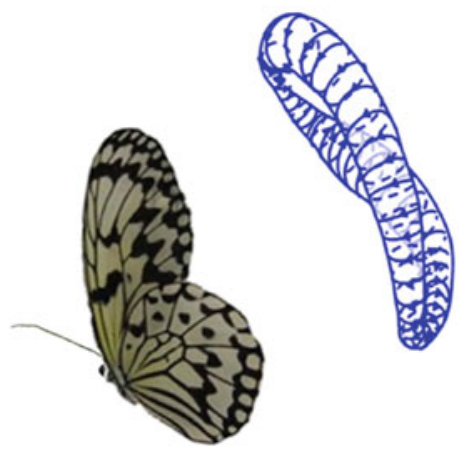

(h) top dead position 
Fig. 17 Pattern diagram of the vortex on the wings of Cynthia cardui for downward flapping motion of the wings

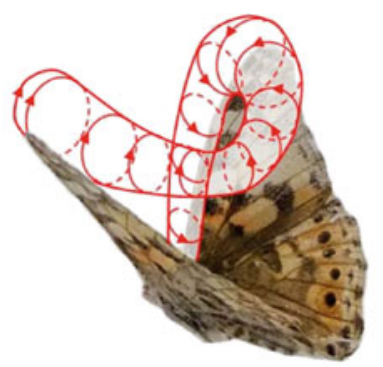

(a) Near top dead position

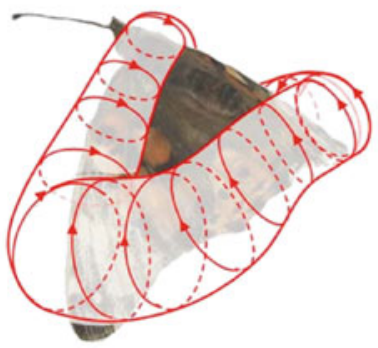

(c) Moving further downward

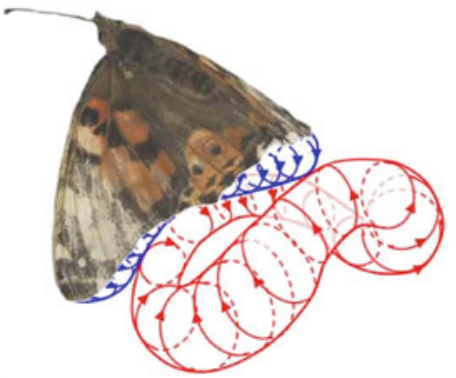

(e) Moving upward

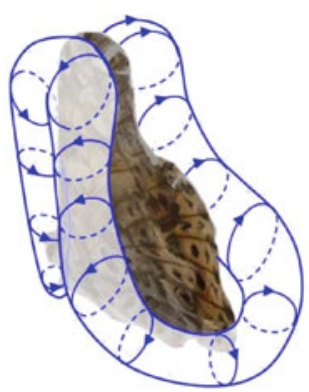

(g) Near top dead position

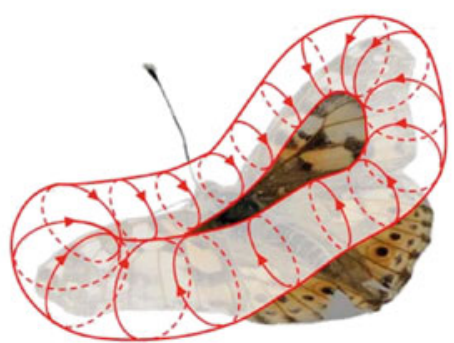

(b) Moving downward

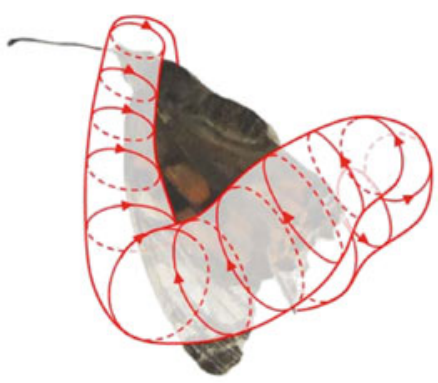

(d) Bottom dead position

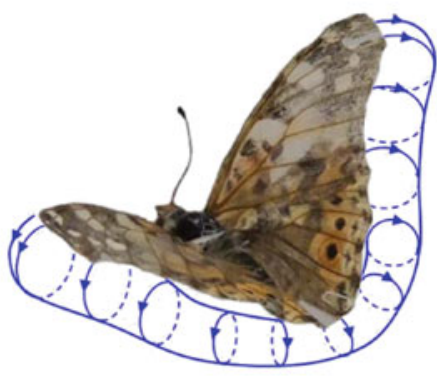

(f) Moving further upward

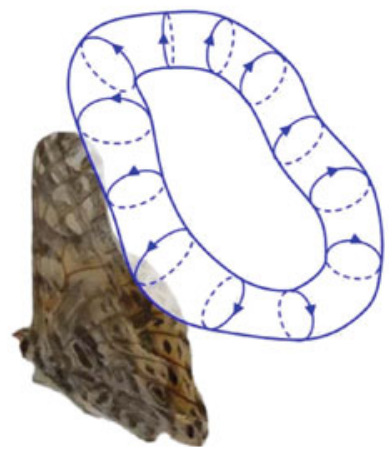

(h) top dead position slightly smaller than that formed in flapping downward. In Idea leuconoe, on the other hand, a large LEV develops near the wing tip because of the large wing. However, the vorticity of the vortex that developed in the spanwise planes was relatively small, and the rear part of the vortex ring elongated. In other words, the dynamic behavior of the vortex ring was found to be affected by the scale of the wings and the flapping frequency.

These vortex rings formed over the butterflies are similar to those described for other insects. Bomphrey et al. (2006) visualized a vortex loop over the wing of a tethered Tobacco Hawkmoth by DPIV. Dickinson and Gotz (1996) 
obtained a vortex loop over the wings of tethered fruit flies, Drosophila melanogaster by qualitative flow visualization and made an animated reconstruction of the vortex loop during one flapping cycle. They also performed instantaneous force measurements of tethered fruit flies and reported that, with each stroke cycle, there is one plateau of high force generation, followed by a period of low force generation, which roughly correlated with the upstroke and downstroke periods. The vortex rings formed over the wings of Cynthia cardui and Idea leuconoe in flapping downward obtained by PIV measurement are very similar to the animated reconstruction of the vortex loop of tethered fruit flies. Moreover, the scale of the vortex ring formed over the wings in flapping upward is different from that in flapping downward. In other words, the dynamic lift produced by the butterfly wing in flapping upward is expected to be different from that in flapping downward because the circulation that develops around the wings differs. Therefore, the obtained results also indicate that there is a period of high force generation followed by a period of low force generation.

In order to quantitatively evaluate the vortices that rolled up from the wings, we evaluate the instantaneous vorticities at 10 wing tip points for six flapping angles in downward and upward flapping by obtaining the PIV measurement, as shown in Fig. 18. Points 1 through 4 show the measurement points for the LEV, and points 5 through 10 show the measurement points for the vortices that developed in the wing spanwise planes. Figure 19a, b show the vorticity on the wing downward and upward flapping, respectively, shown in Fig. 18. The solid black, solid red, and solid blue lines show the results for the downward flapping wing (Fig. 16b), the further downward flapping wing (Fig. 16c), and the bottom dead position (Fig. 16d), respectively, in Fig. 19a, and the broken black, broken red and broken blue lines show the results for the upward flapping wing (Fig. 16e), the further upward flapping wing (Fig. 16f), and the near top dead position (Fig. 16g), respectively, in Fig. 19b. The horizontal and vertical axes show the measurement points and vorticity, respectively.

The vorticity over the wings near the leading edges, as shown by points 3 through 6 , is high and that over the rear part of the wing, as shown by points 8 through 10 , is very low when the wing flaps downward to the bottom dead position. When the wing flaps further downward, the vorticity over the rear part of the wing become highs. Moreover, the vorticity over the wings near the leading edges becomes low, and the vorticity over the trailing edge of the wing, as shown at point 10 , becomes high at the bottom dead position. In other word, the maximum vorticity over the wing moves from the leading edge to the trailing edge with the downward flapping. On the other hand, the vorticity of the LEV decays with downward

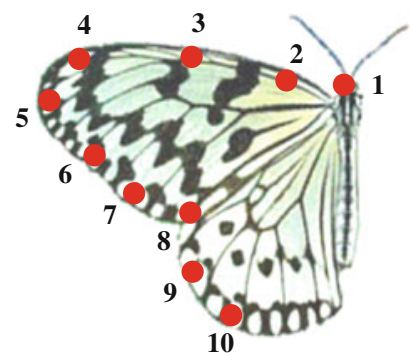

Fig. 18 Measurement points of vorticity on the wings of Idea leuconoe

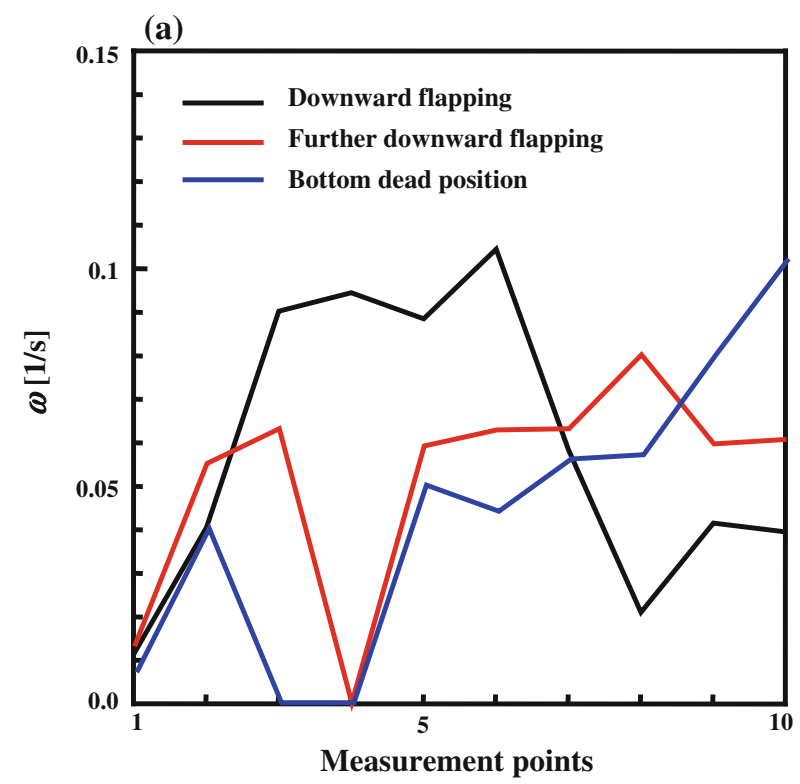

(b)

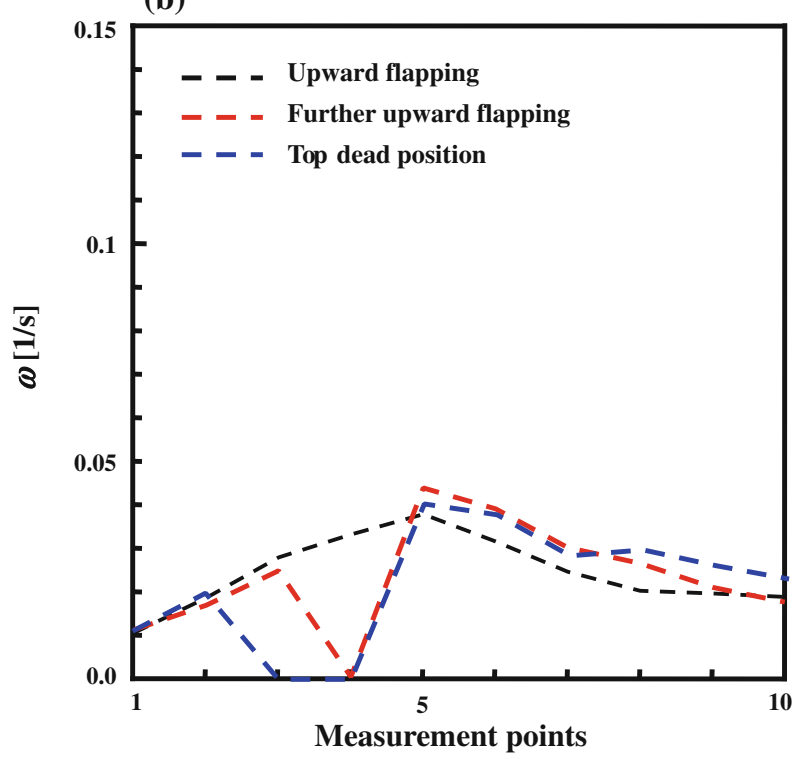

Fig. 19 Vorticity on the wings of Idea leuconoe

flapping. The results shown in Figs. 16 and 19 reveal that the scale and vorticity of the vortex ring develop over the wing while growing from the leading edge toward the 
trailing edge with the downward flapping. When the wing flaps upward, the variation in the vorticity is small. In particular, the vorticity over the rear part of the wing is almost constant with respect to flapping angle. Figure 16 shows that the scale of vortex ring formed in flapping upward is smaller than that formed in flapping downward due to the elastic deformation of the wing. Therefore, the variation of the vorticity of the vortex ring develop over the wing in the upward flapping becomes small.

\section{Conclusions}

A vortex ring is formed over the butterfly wings when the wings flap downward to the bottom dead position. The vortex ring, then, passes completely over the butterfly and grows until reaching the wake at the bottom dead position. The vortex ring is formed over the wings regardless of the type of butterfly, although the scale of the vortex ring varies among butterflies. The scale and vorticity of the vortex ring develop over the wing while growing from the leading edge toward the trailing edge with the downward flapping.

Acknowledgments The authors are grateful to the Mitsubishi Foundation for supporting the present research.

Open Access This article is distributed under the terms of the Creative Commons Attribution License which permits any use, distribution, and reproduction in any medium, provided the original author(s) and the source are credited.

\section{References}

Adrian RJ (1991) Particle-imaging techniques for experimental fluid mechanics. Ann Rev Fluid Mech 23:261-304

Ansari SA, Phillips N, Stabler G, Wilkins PC, Zbikowski R, Knowles K (2009) Experimental investigation of some aspects of insectlike flapping flight aerodynamics for application to micro air vehicles. Exp Fluids 46:777-798

Bomphrey RJ, Lawson NJ, Harding NJ, Taylor GK, Thomas ALR (2005) The aerodynamics of Manduca sexta: digital particle image velocimetry analysis of the leading-edge vortex. J Exp Biol 208:1079-1094

Bomphrey RJ, Lawson NJ, Taylor GK, Thomas ALR (2006) Application of digital particle image velocimetry to insect aerodynamics: measurement of the leading-edge vortex and near wake of a hawkmoth. Exp Fluids 40:546-554
Bomphrey RJ, Taylor GK, Thomas ALR (2009) Smoke visualization of free-flying bumblebees indicates independent leading-edge vortices on each wing pair. Exp Fluids 46:811-821

Dickinson MH, Gotz KG (1996) The wake dynamics and flght forces of the fruit fly Drosophila melanogaster. J Exp Biol 199: 2085-2104

Dickinson MH, Lehmann FO, Sane SP (1999) Wing rotational and aerodynamic basis of insect flight. Science 284(5422): 1954-1960

Fuchiwaki M, Tanaka K (2006) Vortex flow on a butterfly wing. 12th International symposium of flow Vis; Göttingen, September, 12ISFV-156

Fuchiwaki M, Tanaka K (2008) Dynamic behavior of butterfly wing and its vortex flow. 13th international symposium of flow Vis; Nice, July, 13ISFV-103

Fuchiwaki M, Tadatsugu I, Tanaka K (2009) Characteristics of butterfly wing motions and their application to micro flight robot. 48th AIAA aerospace sciences meeting including the new horizons forum and aerospace exposition, Orlando, January

Hart DP (2000) PIV error correction. Exp Fluids 29:13-22

Lawson NJ, Davidson MR (2001) Self-sustained oscillation of a submerged jet in a thin rectangular cavity. J Fluids Struct 15:59-81

Lee JS, Kim JH, Kim C (2008) Numerical study on the unsteady force generation mechanism of insect flapping motion. AIAA J 46(7): $1835-1848$

Marden JH (1987) Maximum production during takeoff in flying animals. J Exp Biol 130:235-258

Senda K, Sawamoto M, Shibahara T, Tanaka T (2004) Study on flapping-of-wings flight of butterfly with experimental measurement. AIAA atmospheric flight mechanics conference. Exhibit; AIAA, Providence, Rhode Island, AIAA-2004-5368

Singh B, Chopra I (2008) Insect-based hover-capable flapping wings for micro air vehicles: experiments and analysis. AIAA J 46(9):2115-2135

Sunada S, Kawachi K, Watanabe I, Azuma A (1993) Performance of a butterfly in take-off flight. J Exp Biol 183:249-277

Tanaka H, Shimoyama I (2010) Forward flight of swallowtail butterfly with simple flapping motion. Bionsp Biomim 5:026003

Tanaka H, Hoshio K, Matsumoto K, Shimoyama I (2005) Flight dynamics of a butterfly-type ornithopter. IEEE/RSJ International conference of intelligent robots and systems; Edmonton, July, pp 2706-2711

Van Den Berg C, Ellington CP (1997) The three-dimensional leadingedge vortex of a 'hovering' model hawkmoth. Phil Trans R Soc Lond B 352:329-340

Yuan L, Gong XS (2008) Three-dimensional flow structures and evolution of the leading-edge vortices on a flapping wing. J Exp Biol 211:1221-1230

Zaeem A, Sunil K, Agrawal K (2006) Design of flapping mechanisms based on transverse bending phenomena in insects. IEEE international conference of robotics and automation; Orlando, pp 2323-2328 